Original Article

\title{
Test-retest reliability of static postural control in people with multiple sclerosis
}

\author{
Maria Mercedes Reguera-García, DPT, MS (PhD candidate) ${ }^{1)}$, \\ Fernanda de Souza-Teixeira, PHE, PhD²), Jose Antonio de Paz Fernández, MD, PhD** \\ 1) School of Health Science, SALBIS Research Group, University of León, Spain \\ 2) School of Physical Education, Departament of Physical Education and Health, Federal University of \\ Pelotas, Brazil \\ 3) Institute of Biomedicina (IBIOMED), University of León: Campus Vegazana s/n 24071, León, Spain
}

\begin{abstract}
Purpose] The purpose of this study was to analyse the intraday test-retest reliability of stabilometric measurements on the Centre of Pressure (CoP) in persons with Multiple Sclerosis. [Subjects and Methods] The measurements were taken with the subject standing on the force platform with both feet together, in four different conditions: eyes open and closed whilst standing on both a rigid surface and then on foam. The measurements were taken on three occasions with intervals of 20 minutes between them. 18 persons with confirmed Multiple Sclerosis (MS), (aged $44.4 \pm 13.3$ years and 3.5 \pm 1.5 EDSS) participated in the study. Relative reliability was measured using the intraclass correlation coefficient (ICC) and the $95 \%$ confidence interval (95\% CI); and the absolute reliability using the coefficient of variation (CV) and standard error of measurement (SEM). [Results] The measurements of the $\mathrm{CoP}$ in all of the variables in each of the four conditions, showed a reliability of good to excellent (ICC $=0.69-0.92)$ in the intraday test-retest. The variables which showed greatest consistency with repetition were X Speed, Y Speed and Side Length. [Conclusion] Given the results it would seem that the best variables for monitoring postural control in persons with MS are Speed on X and Y along with Side Length.

Key words: Balance, Postural control, Stabilometry
\end{abstract}

(This article was submitted Apr. 21, 2017, and was accepted May 24, 2017)

\section{INTRODUCTION}

Multiple Sclerosis (MS) is a demyelinating, neurodegenerative disease of the central nervous system which normally appears in young adults. It is the principal cause of non traumatic disability in young people ${ }^{1)}$. Amongst the symptoms observed are difficulties in moving and postural control with the important implications these have on daily life ${ }^{2)}$. These symptoms are closely related to the severity of the disease ${ }^{3}$.

Postural control is the ability to maintain the projection of the centre of pressure (CoP), within the limits of stability of the supporting base. It is a complex ability, which reflects the motor efficiency to the response of the nervous system with regards to sensory input from the visual, vestibular and somatosensory systems ${ }^{4}$, such that when there is a balance disorder in a patient with MS, it can be difficult to discern to what degree these various afferent and efferent systems are implicated in difficulties with postural control, a complex function whose alteration can cause problems with work and social activities, as well as affecting the psychological wellbeing of the person ${ }^{5}$.

The gold standard measurement for the analysis of postural control is stabilometry, carried out using a force platform with which oscillations of the $\mathrm{CoP}$ are measured, allowing for the detection of minimal displacements with very small sampling frequencies ${ }^{6,7)}$. Stabilometry has become established as a useful diagnostic tool for the prediction of the risk of accidental

*Corresponding author. Jose Antonia de Paz Fernández (E-mail: japazf@unileon.es)

(C2017 The Society of Physical Therapy Science. Published by IPEC Inc.

(c) (1) $\odot$ This is an open-access article distributed under the terms of the Creative Commons Attribution Non-Commercial No Deriva-

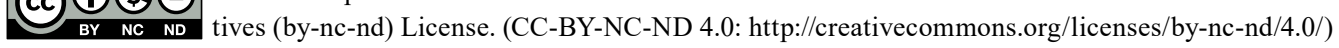




\begin{tabular}{|c|c|c|}
\hline Trial 1 & Trial 2 & Trial 3 \\
\hline $20 \mathrm{~min}$ & $20 \mathrm{~min}$ & \\
\hline Rigid Surface - Eyes Open $(20 \mathrm{~s})$ & Rigid Surface - Eyes Open (20 s) & Rigid Surface - Eyes Open (20 s) \\
\hline 2 minutes & $2 \mathrm{~min}$ & $2 \mathrm{~min}$ \\
\hline Rigid Surface - Eyes Closed (20 seg) & Rigid Surface - Eyes Closed (20 seg) & Rigid Surface - Eyes Closed (20 seg) \\
\hline 2 minutes & $2 \mathrm{~min}$ & $2 \mathrm{~min}$ \\
\hline Foam Surface - Eyes Open $(20 \mathrm{seg})$ & Foam Surface - Eyes Open $(20 \mathrm{seg})$ & Foam Surface - Eyes Open $(20 \mathrm{seg})$ \\
\hline 2 minutes & $2 \mathrm{~min}$ & $2 \mathrm{~min}$ \\
\hline Foam Surface - Eyes Closed $(20 \mathrm{seg})$ & Foam Surface - Eyes Closed (20 seg) & Foam Surface - Eyes Closed (20 seg) \\
\hline
\end{tabular}

Fig. 1. Intraday study design reliability

falls in persons with $\mathrm{MS}^{8)}$. However, the measurement of the displacement of the CoP presents an important variability, both an inter and intra-individual variability which, added to the disorders usually shown by persons with MS, could result in a reduction of reliability for the use of this technique in the clinical monitoring of postural control in this group ${ }^{9)}$. For this reason, it would be useful to know the degree of variability in stabilometry in this group of individuals.

The carrying out of oscillometric evaluations with eyes open and closed, as well as with and without a foam mat, allows us to estimate the contribution of the visual, vestibular and proprioceptive systems in maintaining balance. Thus we decided to analyse the intraday test-retest reliability of the displacement oscillations of the CoP on a rigid surface, on a foam surface, with eyes closed and with eyes open in persons with MS.

\section{SUBJECTS AND METHODS}

The participants in the study were people with a confirmed diagnosis of Relapsing-Remitting MS who had not had an outbreak nor increased worsening in the previous three months and were all capable of standing on two feet and walking without aid for at least 100 minutes, as well as being able to understand and carry out orders. All subjects signed an informed consent form approved by the Ethics committee of the University of León.

On three occasions in the same day, separated by intermissions of 20 minutes, the subjects carried out a protocol consisting of the evaluation of balance while standing barefoot with both feet together. Four different conditions were evaluated: with and without a foam mat, and with eyes open and eyes closed. Each test lasted for 20 seconds in each of the conditions. The subjects were seated between tests for a rest period of 2 minutes (Fig. 1)

The force platform that was used to obtain the results was a Good Balance system (Metitur OY, Jyvaskyla, Finland), with a sample frequency of $50 \mathrm{~Hz}$ with software version GB 300.1.3.20.

The software analyses the following parameters: 1) "Velocity moment" of the CoP (Vel. Moment) (in $\mathrm{mm}^{2} / \mathrm{s}$ ): which is the area covered by the horizontal displacement of the CoP within the support base in a second ${ }^{10,11)}$; 2) "Length of a side of a square" (Side Length) (mm): is the longitude of the side of a square which includes $90 \%$ of the points obtained of the position of the CoP during the 20 seconds of the test; 3) "Mean speed along X axis" (X Speed) (in mm/s): is the average speed of the CoP along the X or mediolateral axis or $(\mathrm{ML})^{9)}$; 4) "Mean speed along Y axis" (Y Speed) (mm/s): is the average speed of the pressure centre along the $\mathrm{Y}$ or anteroposterior axis or $\left.(\mathrm{AP})^{9)} ; 5\right)$ "Distance along $\mathrm{X}$ axis" (X Extension) (mm): is the distance covered by the CoP on the ML axis; 6) "Distance along Y axis" (Y Extension) (mm): is the distance covered by the CoP on the AP axis.

In order to determine the normality of the values of the variables studied, the K-S test was used with the Lilliefors correction.

Reliability was measured using the Intraclass correlation coefficient ICC (2.1). Using the Fleiss criteria, we defined ICC values of less than 0.4 as having poor reliability; 0.40 to 0.75 fair to good reliability; and scores above 0.75 as having excellent reliability ${ }^{12)}$.

To measure absolute reliability, we used the Coefficient Variation (CV). The Atkinson and Alan M. Nevill adaptation was used, which measures the averages of the CV's of each of the subjects for each of their three repetitions ${ }^{13)}$. In the same way, the standard error of measurement (SEM) was used, calculated as the proportion of the deviation not included in the reliability, $\mathrm{SEM}=\mathrm{SD} *(\text { square root of }(1-\mathrm{ICC}))^{14)}$. A level of significance of $\mathrm{p}<0.05$ was established. The statistical analysis packet SPSS 23.0 was used (SPSS, Inc., Chicago, IL, USA).

\section{RESULTS}

7 men and 11 women with MS completed the proposed protocol. The average age was $44.4 \pm 13.3$ years with the average height being $166.6 \pm 10.2 \mathrm{~cm}$ and a weight of $68.1 \pm 11.6 \mathrm{~kg}$. The average time from the initial diagnosis was $9 \pm 5.2$ years. Expanded Disability Status Scale (EDDSS) score was $3.5 \pm 1.5$.

Table 1 shows the average results with S.D. of the CoP as measured in each test and repetition. Table 2 shows the ICC, 
Table 1. Descriptive statistics for MS CoP measures in different sensory condictions by each evaluation moment

\begin{tabular}{|c|c|c|c|}
\hline & Trial 1 & Trial 2 & Trial 3 \\
\hline \multicolumn{4}{|l|}{ Rigid Surface -Eyes Open } \\
\hline Velocity moment $\left(\mathrm{mm}^{2} / \mathrm{s}\right)$ & $34.2 \pm 17.9$ & $38.8 \pm 30.4$ & $38.8 \pm 29.3$ \\
\hline Side Length (mm) & $19.3 \pm 4.9$ & $19.7 \pm 9.1$ & $19.9 \pm 8.1$ \\
\hline X Speed $(\mathrm{mm} / \mathrm{s})$ & $9.9 \pm 4.8$ & $10.2 \pm 5.5$ & $10.4 \pm 4.3$ \\
\hline Y Speed $(\mathrm{mm} / \mathrm{s})$ & $11.5 \pm 5.1$ & $10.7 \pm 4.9$ & $11.1 \pm 5$ \\
\hline X Extension (mm) & $197.6 \pm 93$ & $203.9 \pm 109.1$ & $209.8 \pm 87.5$ \\
\hline Y Extension (mm) & $227.1 \pm 106.2$ & $211.9 \pm 98.9$ & $222.3 \pm 97.9$ \\
\hline \multicolumn{4}{|l|}{ Rigid Surface -Eyes Closed } \\
\hline Velocity moment $\left(\mathrm{mm}^{2} / \mathrm{s}\right)$ & $108.9 \pm 120.2$ & $109.1 \pm 113.5$ & $81.2 \pm 80.1$ \\
\hline Side Length $(\mathrm{mm})$ & $29.3 \pm 13.6$ & $31.4 \pm 13.9$ & $27.7 \pm 13.3$ \\
\hline X Speed $(\mathrm{mm} / \mathrm{s})$ & $18.4 \pm 11.1$ & $18.2 \pm 11.8$ & $15.6 \pm 8.3$ \\
\hline Y Speed $(\mathrm{mm} / \mathrm{s})$ & $20.9 \pm 12.2$ & $19.4 \pm 11$ & $16.9 \pm 8.2$ \\
\hline X Extension (mm) & $367.6 \pm 223.6$ & $363 \pm 237.7$ & $311 \pm 165.8$ \\
\hline Y Extension (mm) & $415.8 \pm 242.3$ & $389.1 \pm 217.9$ & $337.5 \pm 162.5$ \\
\hline \multicolumn{4}{|l|}{ Foam Surface -Eyes Open } \\
\hline Velocity moment $\left(\mathrm{mm}^{2} / \mathrm{s}\right)$ & $61.8 \pm 45.2$ & $49.2 \pm 27.2$ & $53.9 \pm 30.8$ \\
\hline Side Length $(\mathrm{mm})$ & $25.8 \pm 10.2$ & $23.1 \pm 7.1$ & $24.6 \pm 8.6$ \\
\hline $\mathrm{X}$ Speed $(\mathrm{mm} / \mathrm{s})$ & $17.6 \pm 13.5$ & $12.6 \pm 4.9$ & $13.3 \pm 5.7$ \\
\hline Y Speed $(\mathrm{mm} / \mathrm{s})$ & $14.5 \pm 5.51$ & $12.33 \pm 3.74$ & $13.1 \pm 5.1$ \\
\hline X Extension (mm) & $197.6 \pm 93$ & $203.9 \pm 109.1$ & $209.8 \pm 87.5$ \\
\hline Y Extension (mm) & $227.1 \pm 106.2$ & $211.9 \pm 98.9$ & $222.3 \pm 97.9$ \\
\hline \multicolumn{4}{|l|}{ Foam Surface -Eyes Closed } \\
\hline Velocity moment $\left(\mathrm{mm}^{2} / \mathrm{s}\right)$ & $105.3 \pm 87.3$ & $87.9 \pm 70.2$ & $80.6 \pm 53.5$ \\
\hline Side Length (mm) & $30.6 \pm 11.8$ & $28.6 \pm 10.6$ & $28.7 \pm 9.7$ \\
\hline$X$ Speed $(\mathrm{mm} / \mathrm{s})$ & $18.6 \pm 8.6$ & $17.3 \pm 8.3$ & $16.3 \pm 6.8$ \\
\hline Y Speed $(\mathrm{mm} / \mathrm{s})$ & $19.3 \pm 8$ & $17.5 \pm 6.9$ & $16.5 \pm 6.2$ \\
\hline $\mathrm{X}$ Extension (mm) & $372.5 \pm 171.9$ & $345.9 \pm 166.9$ & $326.5 \pm 135.1$ \\
\hline Y Extension $(\mathrm{mm})$ & $384.1 \pm 161.4$ & $348.8 \pm 136.8$ & $331.3 \pm 122.5$ \\
\hline
\end{tabular}

Side Length: length of a side of a square; X Speed: Mean speed along X axis; Y Speed: Mean speed along Y axis; X Extension: Distance along $\mathrm{X}$ axis; Y Extension: Distance along $\mathrm{Y}$ axis

the $95 \%$ IC, CV and SEM.

We observed relative reliability values from reasonable to excellent in all variables of each test conducted on three different occasions ( $\mathrm{ICC}=0.92-0.69$ ). The $\mathrm{CV}$ of the tests varied from $13.4 \%$ to $18.9 \%$ in all variables except for velocity moment which exceeded said values. The SEM of all the variables of the repeated tests ranged from between 1.5 and 94.2. The variables which obtained the best result for reliability, regardless of the test, were speed along X and Y and Side Length. These variables obtained an SEM between 1.5 and 6.3 and the CV between 13.4 and 18.9.

\section{DISCUSSION}

This study evaluated the reliability of the oscillometric variables of movement of the CoP in 3 repetitions carried out on the same day on a firm surface, a soft (foam) surface, with eyes open and eyes closed, in persons with MS. The main discovery was a relative reliability of good to excellent $(\mathrm{ICC}=0.69-0.92)$ in the tests with eyes both open and closed on a firm surface, as well as with eyes both open and closed whilst standing on a foam mat. The greatest absolute reliability amongst the repeated tests was obtained from the average speed along $\mathrm{X}$ and $\mathrm{Y}$ variables and also from Side Length, which gave a value lower than that of CV and SEM.

The CoP measurements studied gave different degrees of reliability, both relative and absolute, given that each test measures a different sensorial condition ${ }^{15}$. The resulting data reveal higher ICC levels on a firm surface than on foam. These results may be explained by the fact that postural strategies on a foam mat vary due to compensatory movements ${ }^{16)}$. Other authors argue that the strategies used to attain postural control are the same as those used to maintain movement stability, which could explain why our values have similar reliability results between rigid surface and mat ${ }^{17)}$. On the other hand, 
Table 2. Test-retest reliability of CoP Measures in different sensory condictions of multiple sclerosis individuals $(n=18)$

\begin{tabular}{|c|c|c|c|c|}
\hline & ICC & $95 \% \mathrm{CI}$ & $\mathrm{CV}(\%)$ & SEM \\
\hline \multicolumn{5}{|l|}{ Rigid Surface -Eyes Open } \\
\hline Velocity moment $\left(\mathrm{mm}^{2} / \mathrm{s}\right)$ & 0.69 & $0.5-0.9$ & 29.3 & 14.4 \\
\hline Side Length (mm) & 0.74 & $0.5-0.9$ & 16.7 & 3.8 \\
\hline X Speed $(\mathrm{mm} / \mathrm{s})$ & 0.81 & $0.6-1.0$ & 18.6 & 2.1 \\
\hline Y Speed $(\mathrm{mm} / \mathrm{s})$ & 0.92 & $0.8-1.0$ & 13.4 & 1.5 \\
\hline X Extension (mm) & 0.81 & $0.6-0.9$ & 17.6 & 42.4 \\
\hline Y Extension (mm) & 0.92 & $0.8-1.0$ & 13.4 & 28.6 \\
\hline \multicolumn{5}{|l|}{ Rigid Surface -Eyes Closed } \\
\hline Velocity moment $\left(\mathrm{mm}^{2} / \mathrm{s}\right)$ & 0.83 & $0.7-0.9$ & 30.4 & 43 \\
\hline Side Length (mm) & 0.81 & $0.6-0.9$ & 15.9 & 6 \\
\hline X Speed $(\mathrm{mm} / \mathrm{s})$ & 0.84 & $0.7-0.9$ & 18.7 & 4.1 \\
\hline Y Speed $(\mathrm{mm} / \mathrm{s})$ & 0.80 & $0.6-0.9$ & 17.1 & 4.7 \\
\hline X Extension (mm) & 0.85 & $0.7-0.9$ & 18.7 & 81 \\
\hline Y Extension (mm) & 0.79 & $0.6-0.9$ & 17.1 & 94.2 \\
\hline \multicolumn{5}{|l|}{ Foam Surface -Eyes Open } \\
\hline Velocity moment $\left(\mathrm{mm}^{2} / \mathrm{s}\right)$ & 0.69 & $0.4-0.8$ & 28.7 & 19.2 \\
\hline Side Length (mm) & 0.69 & $0.4-0.8$ & 18.2 & 4.8 \\
\hline X Speed $(\mathrm{mm} / \mathrm{s})$ & 0.69 & $0.4-0.7$ & 17.9 & 6.3 \\
\hline Y Speed $(\mathrm{mm} / \mathrm{s})$ & 0.70 & $0.5-0.8$ & 16.1 & 2.6 \\
\hline X Extension (mm) & 0.73 & $0.5-0.9$ & 20.3 & 50.4 \\
\hline Y Extension (mm) & 0.70 & $0.5-0.9$ & 16.1 & 55 \\
\hline \multicolumn{5}{|l|}{ Foam Surface -Eyes Closed } \\
\hline Velocity moment $\left(\mathrm{mm}^{2} / \mathrm{s}\right)$ & 0.69 & $0.4-0.8$ & 28.1 & 39.2 \\
\hline Side Length (mm) & 0.80 & $0.6-0.9$ & 13.6 & 4.8 \\
\hline X Speed $(\mathrm{mm} / \mathrm{s})$ & 0.79 & $0.6-0.9$ & 16.7 & 3.6 \\
\hline Y Speed $(\mathrm{mm} / \mathrm{s})$ & 0.70 & $0.5-0.9$ & 18.9 & 3.8 \\
\hline X Extension (mm) & 0.79 & $0.6-0.9$ & 16.7 & 72.6 \\
\hline Y Extension $(\mathrm{mm})$ & 0.70 & $0.5-0.9$ & 18.9 & 76.7 \\
\hline
\end{tabular}

Side Length: length of a side of a square; X Speed: Mean speed along X axis; Y Speed: Mean speed along Y axis; X Extension: Distance along X axis; Y Extension: Distance along Y axis; ICC: intraclass correlation coefficient; 95\% CI: confidence interval; CV: coefficient of variation; SEM: standard error of measurement

other authors propose the combined use of force platforms with a tri dimensional movement analysis system in order to establish a permissible range of balance to get a deeper understanding of the reliability of tests done on a mat ${ }^{18)}$. Although we were unable to find studies with the same protocol and platform, we did find intraday studies with similar protocols (eyes open and closed on a firm surface and on foam) in elderly persons, such as the study carried out by Benvenuti et al ${ }^{18)}$. In this study, higher values were obtained for reliability for the velocity variable for the centre of gravity and for the CoP $(\mathrm{ICC}=0.76-0.74)$. We believe this is possibly due to the fact that postural control is less affected by aging than it is by MS, and what's more, variability amongst the elderly tends to be less than that of patients with MS due to the varying degrees and types of effects. On the other hand, in the Alahmari et al. study ${ }^{19)}$ we found a protocol that was similar to ours, in which they added an evaluation with a sway-referenced visual surround, obtaining similar results in the speed variable $($ ICC $=0.61-0.85)$. We believe this is due to the similarity between the symptoms of the vestibular alteration study group and ours. In respect of the extension on both axes variable we found the Brenton-Rule et al. study ${ }^{20)}$ which shows results of excellent intraday reliability with an interval of an hour between each evaluation ( ICC $=0.89-0.92$ ), carried out with eyes open and closed on a firm surface in elderly persons with ICC values that were very similar to the results from our study with the same conditions and variables studied.

With regards to the methodology we employed, we found that studies which involved an average of 3 measurements of the CoP on every repetition on a firm surface with eyes open, obtained ICC values of speed along X and Y ICC that were slightly higher than ours in the elderly (ICC $=0.96 / 0.96)$ and in young adults $(\mathrm{ICC}=0.96 / 0.96)$. This may be because the number of measurements and repetitions affects the degree of reliability ${ }^{6,21)}$. Concerning time elapsed between repetitions; we didn't find reliability studies on the same day with the Good Balance platform in any group. However, we checked test-retest reli- 
ability values on persons with MS between days which showed an inferior reliability in comparison to our data, as was to be expected ${ }^{6}$. Paltamaa et al. ${ }^{11)}$ studied the test-retest in MS with a time difference of a week, using the same platform as ours on a firm surface with eyes open and closed, showing lower repeatability values in the velocity moment in ICC, SEM and $\mathrm{CV}$. By contrast, researchers such as Wajda et al. ${ }^{22}$ showed reliability in MS over three month test-retest reliability of CoP motion during standing balance in individuals with MS obtaining results that were very similar to those we obtained from our ICC results from the variable of speed along both axes with eyes open and closed on a firm surface. This suggests to us that adding a dual task to the measurements as done by Wadja et al. ${ }^{22}$ might increase the attention of the individual with MS and thus its reliability in time ${ }^{4)}$.

Another factor which may influence the reliability of the tests is the range of sampling frequencies of the platforms. Our study was conducted at $50 \mathrm{~Hz}$, whilst other studies have used a frequency of $100^{23)}$ or $200 \mathrm{~Hz}^{24)}$.

With regards to the $\mathrm{CV}$ of the study, this varied between $13.4 \%$ and $18.9 \%$, except with velocity moment which exceeds said values (28.1\% to $30.4 \%)$. Some authors suggest that the CV may vary depending on the study or the study group. But the basic premise is that values above $30 \%$ should not be considered, as was the case with our study in all cases ${ }^{25}$. By contrast, other authors use the stricter criteria of a maximum of $15 \%$ for the $\mathrm{CV}$ for repeated force platform tests ${ }^{26)}$. In any event, the CV should be considered with caution because it should only be applied in the case of normal distribution of all of the variables ${ }^{13)}$. Our results didn't have normal distribution in all variables, the same as occurred in the reliability study on persons with MS conducted by Paltamaa el $\mathrm{al}^{11)}$.

As a measure of absolute reliability, the SEM of the current study shows that the most traditional variables, such as speed along the $\mathrm{X}$ or $\mathrm{Y}$ axis and side length have the best values, being the lowest values in these variables in all tests carried out. As such, they should be considered as important reference variables for the monitoring of balance in these patients. Other studies which evaluated the intraday SEM, obtained lower values of reliability in variables related to speed but the data contradict results obtained for $\mathrm{CoP}$ displacement during the test ${ }^{19,24)}$. We think that this may partly be due to the methods used to calculate the area; some use the elliptical form whilst others use the square, although all usually consider $90 \%$ of the points obtained during the test.

The current study showed that postural control measurements taken from the Good Balance System ${ }^{\circledR}$ platform gave a reliability of good to excellent with eyes open and closed, on a firm surface and on a foam mat (ICC $\geq 0.69$ ), but it has its limitations. The limitations were a scarcity of reliability studies on persons with MS to compare with our data, as well as an abnormal distribution of the sample which could affect the interpretation of the absolute reliability. Perhaps, and it would need further studies, average measurements from three repetitions along with induction sessions would increase the reliability values.

The intraday reliability of the CoP in persons with MS, on a firm surface with eyes open and/or closed, and on a foam surface with eyes open and/or closed is from good to excellent in all variables of the protocol that was carried out whilst standing with both feet together. The most reliable variables for the monitoring of postural control in patients with MS are Speed along $\mathrm{X}$ and $\mathrm{Y}$ as well as Side Length. In future studies it would be advisable to verify these results and evaluate if the intraday reliability of the CoP would increase with the average of more measurements in each session.

\section{ACKNOWLEDGEMENT}

Funding/Support: This study was based on work supported by the Consejería de Sanidad y Consumo, Government of Castilla y León, Spain.

\section{REFERENCES}

1) Hunter SF: Overview and diagnosis of multiple sclerosis. Am J Manag Care, 2016, 22: s141-s150. [Medline]

2) NilsagÅrd Y, Lundholm C, Denison E, et al.: Predicting accidental falls in people with multiple sclerosis—a longitudinal study. Clin Rehabil, 2009, 23: 259-269. [Medline] [CrossRef]

3) Corporaal SH, Gensicke H, Kuhle J, et al.: Balance control in multiple sclerosis: correlations of trunk sway during stance and gait tests with disease severity. Gait Posture, 2013, 37: 55-60. [Medline] [CrossRef]

4) Shumway-Cook A, Woollacott MH: Motor control: translating research into clinical practice. Philadelphia: Lippincott \&Williams, 2012.

5) Ponzio M, Tacchino A, Zaratin P, et al.: Unmet care needs of people with a neurological chronic disease: a cross-sectional study in Italy on Multiple Sclerosis. Eur J Public Health, 2015, 25: 775-780. [Medline] [CrossRef]

6) Ruhe A, Fejer R, Walker B: The test-retest reliability of centre of pressure measures in bipedal static task conditions-a systematic review of the literature. Gait Posture, 2010, 32: 436-445. [Medline] [CrossRef]

7) Rodríguez Fernández C, Mata Zubillaga D, Rodríguez Fernández LM, et al.: Valoración de la coordinación y el equilibrio en niños prematuros. An Pediatría, 2016, 85: 86-94. [CrossRef]

8) Prosperini L, Fortuna D, Giannì C, et al.: The diagnostic accuracy of static posturography in predicting accidental falls in people with multiple sclerosis. Neurorehabil Neural Repair, 2013, 27: 45-52. [Medline] [CrossRef]

9) Huisinga JM, Yentes JM, Filipi ML, et al.: Postural control strategy during standing is altered in patients with multiple sclerosis. Neurosci Lett, 2012, 524: 
124-128. [Medline] [CrossRef]

10) Ha H, Cho K, Lee W: Reliability of the good balance system ${ }^{(\circledR)}$ for postural sway measurement in poststroke patients. J Phys Ther Sci, 2014, 26: 121-124. [Medline] [CrossRef]

11) Paltamaa J, West H, Sarasoja T, et al.: Reliability of physical functioning measures in ambulatory subjects with MS. Physiother Res Int, 2005, 10: 93-109. [Medline] [CrossRef]

12) Shrout PE, Fleiss JL: Intraclass correlations: uses in assessing rater reliability. Psychol Bull, 1979, 86: 420-428. [Medline] [CrossRef]

13) Atkinson G, Nevill AM: Statistical methods for assessing measurement error (reliability) in variables relevant to sports medicine. Sports Med, 1998, 26: 217-238. [Medline] [CrossRef]

14) Dudek FJ: The continuing misinterpretation of the standard error of measurement. Psychol Bull, 1979, 86: 335-337. [CrossRef]

15) Dickin DC, Clark S: Generalizability of the sensory organization test in college-aged males: obtaining a reliable performance measure. Clin J Sport Med, 2007, 17: 109-115. [Medline] [CrossRef]

16) Horak FB: Clinical assessment of balance disorders. Gait Posture, 1997, 6: 76-84. [CrossRef]

17) Feldman AG: The relationship between postural and movement stability. Adv Exp Med Biol, 2016, 957: 105-120. [Medline] [CrossRef]

18) Benvenuti F, Mecacci R, Gineprari I, et al.: Kinematic characteristics of standing disequilibrium: reliability and validity of a posturographic protocol. Arch Phys Med Rehabil, 1999, 80: 278-287. [Medline] [CrossRef]

19) Alahmari KA, Marchetti GF, Sparto PJ, et al.: Estimating postural control with the balance rehabilitation unit: measurement consistency, accuracy, validity, and comparison with dynamic posturography. Arch Phys Med Rehabil, 2014, 95: 65-73. [Medline] [CrossRef]

20) Brenton-Rule A, Mattock J, Carroll M, et al.: Reliability of the TekScan MatScan ${ }^{\circledR}$ system for the measurement of postural stability in older people with rheumatoid arthritis. J Foot Ankle Res, 2012, 5: 21. [Medline] [CrossRef]

21) Demura S, Kitabayashi T, Aoki H: Body-sway characteristics during a static upright posture in the elderly. Geriatr Gerontol Int, 2008, 8: 188-197. [Medline] [CrossRef]

22) Wajda DA, Motl RW, Sosnoff JJ: Three-month test-retest reliability of center of pressure motion during standing balance in individuals with multiple sclerosis. Int J MS Care, 2016, 18: 59-62. [Medline] [CrossRef]

23) Wheat JS, Haddad JM, Scaife R: Between-day reliability of time-to-contact measures used to assess postural stability. Gait Posture, 2012, 35: 345-347. [Medline] [CrossRef]

24) Golriz S, Hebert JJ, Foreman KB, et al.: The reliability of a portable clinical force plate used for the assessment of static postural control: repeated measures reliability study. Chiropr Man Therap, 2012, 20: 14. [Medline] [CrossRef]

25) Harringe ML, Halvorsen K, Renström P, et al.: Postural control measured as the center of pressure excursion in young female gymnasts with low back pain or lower extremity injury. Gait Posture, 2008, 28: 38-45. [Medline] [CrossRef]

26) Di Berardino F, Filipponi E, Barozzi S, et al.: The use of rubber foam pads and "sensory ratios" to reduce variability in static posturography assessment. Gait Posture, 2009, 29: 158-160. [Medline] [CrossRef] 